\title{
Análisis de la Reforma Educativa en México, desde la perspectiva de Educación Física
}

\author{
Analysis of the Educational Reform in México, \\ from the perspective of the physical education subject
}

* Adrián Alonso Ramírez García, ** Diana Eunice Chel Hoil

Ramírez, A., Chel, D. (2019). Análisis de la Reforma Educativa en México, desde la perspectiva de Educación Física. Revista Ciencias de la Actividad Física UCM, No 20(2), julio-diciembre, 1-17.

DOI: http://doi.org/10.29035/rcaf.20.2.2

\section{RESUMEN}

El propósito de este trabajo es analizar el Nuevo Modelo propuesto recientemente en México desde la perspectiva de la Educación Física, dada la importancia de la asignatura en proceso formativo de los alumnos de la educación básica. El análisis de la documentación se realizó utilizando la técnica $\vee$ de Gowin, que como estrategia permitió analizar críticamente el nuevo modelo educativo. El currículo plantea el enfoque competencial desarrollando el pensamiento complejo de los educandos, que es la base para que gestionen sus conocimientos. Este cambio de programa pudiera ser una gran oportunidad para que los educadores físicos cambiaran a estilos de enseñanza que permita la participación activa y reflexiva de los alumnos. Las prácticas educativas de los docentes tienen una gran relación con su formación inicial y la actualización en activo, por lo que es importante atender el proceso formativo y de actualización, ya que son las mismas autoridades las que permiten que se sigan utilizando enfoques tradicionales en lugar de los propuestos en la actualidad.

\section{PALABRAS CLAVE}

Educación física, reforma educativa, enfoque pedagógico, formación inicial, actualización en activo.

* Facultad de Ciencias de la Actividad Física. UACH. Servicios Educativos del Estado de Chihuahua.

** Facultad de Ciencias de la Actividad Física. UACH. 


\section{ABSTRACT}

The purpose of this work is to analyze the New Educational Model proposed recently in Mexico from the physical education perspective, given the importance of the subject in the formative process of elementary education students. The analysis of the documentation was carried out using Gowin's $\checkmark$ technique, which, as a strategy, allowed for a critical analysis of the new educational model. The curriculum raises the competence approach by developing the learner's complex thinking, which is the basis for managing their knowledge. This change in the program could be a great opportunity for physical educators to change teaching styles that allow the active and reflexive participation of students. The teachers' educational practices have a great relationship with the initial training and active updating, so it is important to attend newtraining and updating process since they are the same authorities that continuously use traditional approaches instead of currently proposed ones. The teacher is the one who organizes the learning and interacts directly with learners.

\section{Key words}

Physical education, educational reform, pedagogical approach, initial formation, active up.

\section{Introducción}

Las reformas educativas son un tema de gran interés en el mundo; en las últimas décadas existe un rápido aumento y alcance del conocimiento, la toma de conciencia de que el objetivo principal de un currículo debería ser jóvenes "competentes" y no únicamente de estudiantes capaces de memorizar, y que el currículo deberá estar vinculado al conocimiento adquirido fuera de la escuela y al aprendizaje posterior a ésta, por lo que se cuestionan cada vez más los currículos tradicionales determinados por las autoridades centrales (UNESCO,
2017). Es esencial analizar el Nuevo Modelo propuesto desde la perspectiva de la Educación Física (EF), dada la importancia de la asignatura en el proceso formativo de los alumnos de la educación básica.

La propuesta inicial de cambiar el nombre de la asignatura de "Educación Física" por "Desarrollo Corporal y Salud" fue motivo de fuerte resistencia de los profesionales del área; lamentablemente no lo ha sido el análisis acerca de temas fundamentales de la reforma educativa, como el 
currículo del nuevo modelo y sus implicaciones pedagógicas, didácticas y del trabajo docente, así como de los retos para las escuelas formadoras y autoridades educativas sobre la formación inicial y en activo.

Figueras, Capllonch, Blázquez, \& Monzonís (2016) mencionan que a partir de la implementación del enfoque de competencias en los currícuIos, en EF se realizan investigaciones que analizan la necesidad de incluir las competencias motrices en las competencias claves, para enriquecer las propuestas del modelo competencial. Asimismo, Coutín, Gastélum, \& Guedea (2018), en su estudio dicen que los currículos de EF deben ser proyectos integrales, flexibles, que abarquen los aprendizajes propios de la asignatura, pero que se vinculen con las demás áreas de la educación de los estudiantes. Betancur-Agudelo, López-Ávila \& Arcila-Rodríguez (2018) explican que los procesos de formación que se están desarrollando en la clase de EF no poseen mucha relevancia, pues la sesión es un espacio en donde los docentes se preocupan más por generar disciplina y control, acciones que se alejan de los propósitos educativos de los planes y programas actuales.

En cuanto a la formación profesional, las autoridades elaboran documentos que permitan dar seguimiento a la formación continua, como es el caso de la "Estrategia Nacional 2018", aunque en muchos casos, estas me- didas son percibidas por los docentes como apoyos que solo incrementan su trabajo docente.

\section{Antecedentes}

En los últimos años en México se lleva cabo una reforma de la educación básica, con el argumento de que los currículos anteriores privilegiaban los contenidos de aprendizajes cognitivos y descuidaban otros aspectos como el psicomotor y afectivo-social, no siendo congruente con las necesidades de los educandos, por ello, se plantea la necesidad de reformar los programas tomando en cuenta criterios internacionales modernos. Opertti (2017) en la Agenda Educativa 2030, asevera que los grandes cambios por el inicio de la llamada cuarta revolución industrial hacen necesario ajustar los conceptos educativos que se han realizado en las últimas décadas. Una revolución industrial que se caracteriza por la presencia universal de Internet sobre la forma móvil con captores que son crecientemente más pequeños, más potentes y baratos.

La propuesta educativa, explícita o implícitamente, se sustenta en diálogo, con todas sus contradicciones y conflictos en torno a una triada: sociedad, política y educación, con un equilibrio entre las tres, y que permita realizar reformas adecuadas a nuestros tiempos (Agenda Educativa 2030). 
Casanova, Díaz-Barriga, Loyo, Rodríguez \& Rueda (2017) analizan desde la investigación educativa la reforma educativa, he aquí algunos de sus comentarios:

Casanova dice: "Aunque resulta difícil aludir a las fortalezas de un modelo educativo que surge en el último tercio del sexenio, pero que positivamente se hacen explícitos algunos planteamientos pedagógicos que hasta ahora habían aparecido de manera velada en el discurso gubernamental" (p. 194). Díaz-Barriga comenta: "Quizá una ventaja que tiene la presentación del nuevo modelo es que se han escuchado opiniones de diversos grupos: empresarios, profesores, aunque con una significativa ausencia de investigadores educativos. La autoridad educativa no tiene claro cómo atenderlos" (p. 195). Rueda argumenta: "La propuesta contenida en el nuevo modelo educativo, además de ser poco clara, está disociada de la formación de los maestros" (p. 202).

Haciendo una revisión de los enfoques de los últimos cuatro programas de EF en nuestro país, han sido acordes con las posturas a nivel mundial. Tabla 1.

\section{Tabla 1}

Enfoques pedagógicos de los programas de educación física.

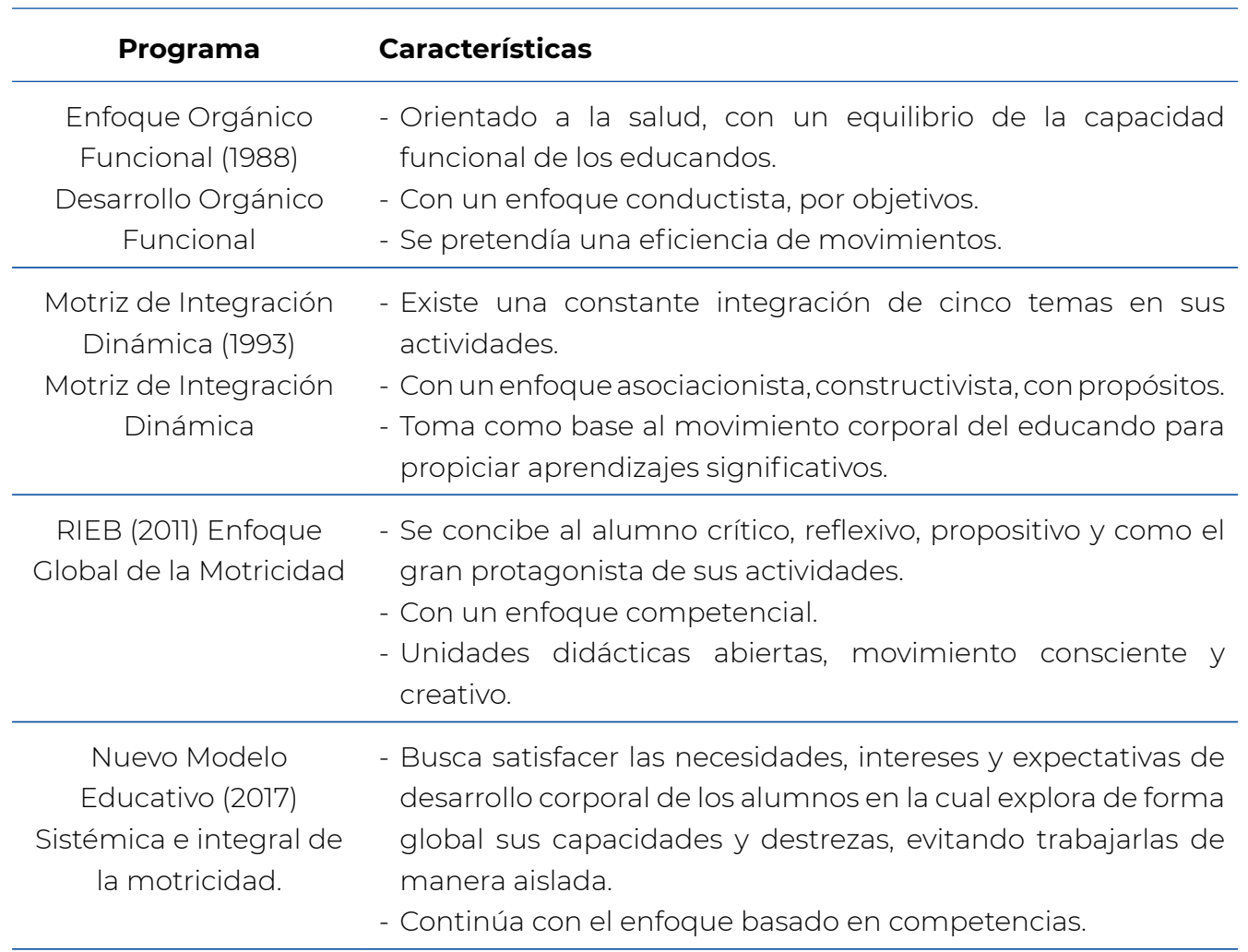


Sería recomendable reflexionar sobre los enfoques pedagógicos y didácticos, ya que es primordial que los profesionales de EF, docentes, supervisores, asesores técnicos y autoridades los tengan muy claros. Lucio (1992) afirma que la pedagogía se pregunta cómo educar y se expresa en los programas educativos y se concreta en la escuela por los profesores, mientras la didáctica se pregunta cómo enseñar, se expresa en un currículo y se concretiza en el aula.

\section{Desarrollo}

\section{Metodología}

Para la revisión y análisis del Programa 2017 se utilizó la técnica $\vee$ de Gowin, ya que, como modelo indagatorio a través de un diagrama -en forma de $\vee$ - se visualiza cómo se estructura el conocimiento (Herrera San Martín, 2012), en este caso, como un instrumento que permita analizar críticamente un proceso educativosocial, como lo es una reforma educativa, y adecuándolo a las necesidades y características del presente trabajo. El proceso de revisión y análisis se puede visualizar en la Figura 1. 


\begin{tabular}{|c|c|c|}
\hline ZT & ZT & ZM \\
\hline $\begin{array}{l}\text { Zona teórica } \\
\text { Análisis de Programa } \\
\text { 2017 } \\
\text { 5.Programa 2017, } \\
\text { currículo y enfoque } \\
\text { - Formación inicial y en } \\
\text { activo. } \\
\text { - Salud, tema transversal. } \\
\text { - Planeación y evaluación. } \\
\text { - Interacción de } \\
\text { capacidades, habilidades } \\
\text { y destrezas. } \\
\text { - Intervención pedagógica. } \\
\text { Enfoque pedagógico: } \\
\text { 3. Reforma educativa } \\
\text { - Nuevo modelo educativo, } \\
\text { reformas, currículos, } \\
\text { enfoques en educación } \\
\text { física. }\end{array}$ & $\begin{array}{l}\text { 1. Modelo educativo } 2017 \\
\text { Zona de problemas } \\
\text { 2. ¿Cómo implementar } \\
\text { el programa } 2017 \text { en } \\
\text { educación física? }\end{array}$ & $\begin{array}{l}\text { Zona de método } \\
\text { Análisis con base en referentes } \\
\text { 6. Desarrollo y discusión } \\
\text { - Escuelas formadoras y } \\
\text { autoridades } \\
\text { educativas. } \\
\text { - La salud en EF. } \\
\text { - Planeación y evaluación } \\
\text { - Patrones básicos de movimiento. } \\
\text { - Competencias, pensamiento } \\
\text { complejo enfoque sistémico. } \\
\text { - Metodología V de Gowin, como } \\
\text { análisis crítico. } \\
\text { 4. Introducción y antecedentes } \\
\text { - Antecedentes de enfoques de los } \\
\text { últimos programas de educación } \\
\text { física. } \\
\text { - Relevancia de la EF en currículo. } \\
\text { - Reformas globales y en el país; } \\
\text { agendas educativas prospectivas } \\
\text { y la investigación educativa. }\end{array}$ \\
\hline
\end{tabular}

Figura 7. Análisis y revisión del modelo educativo con la técnica V Gowin.

\section{Programa 2017: currículo, enfoques, formación docente}

El programa establece que "La Educación Física es una forma de intervención pedagógica que contribuye a la formación integral de niñas, niños y adolescentes al desarrollar su motricidad e integrar su corporeidad" (Secretaría de Educación Pública, 2017, p. 161), una asignatura eminentemente práctica que tiene como finalidad que los alumnos construyan competencias motrices.

Hay una continuidad del anterior programa -2011- en que la EF estaba ubicada en el campo de formación Desarrollo Personal y la Convivencia, junto con educación cívica y artística, mientras que en el programa actual se ubica en el área de Desarrollo Per- 
sonal y Social, junto con educación artística y socioemocional. En el nuevo modelo permanece el enfoque de desarrollo de competencias, relacionando lo aprendido en la escuela y aplicandolo en sus actividades cotidianas. El enfoque pedagógico de la EF se establece de una forma bastante explicita que permite tanto definir las intenciones formativas, como encauzar la motricidad y corporeidad de los alumnos, planteado a través de cuatro categorías o elementos:

\section{La intervención pedagógica de la EF}

Se da continuidad al enfoque global de la motricidad del programa anterior, denominado global de la motricidad que buscaba desarrollar competencias educativas y para la vida, promoviendo una comprensión de la motricidad creativa que contemple la complejidad del quehacer humano, planteando ahora una orientación sistémica e integral de la motricidad. Se dice sistémico pues, de manera organizada, pretende satisfacer en los estudiantes sus intereses y expectativas de su corporeidad y movimiento, siempre compartiendo colectivamente, e integral, pues los alumnos exploran de manera global sus habilidades y destrezas poniendo especial atención, y tomando en cuenta, el crecimiento y maduración.

\section{La interacción de las capacidades, habilidades y destrezas que pro- mueve la EF}

Cuando los alumnos están realizando sus actividades en la sesión de EF están presentes tres factores: los propósitos que se pretenden, en qué condiciones las han de realizar y las capacidades de cada alumno. Para favorecer la corporeidad, motricidad y creatividad, los alumnos utilizan sus capacidades perceptivo-motrices, socio-motrices, y físico-motrices, así como sus habilidades y destrezas motrices y los docentes, con su intervención, deben proponer actividades en que se exploren y busquen soluciones, ya sea de manera individual o colectiva.

\section{Planificación y evaluación en Educación Física}

La intervención docente en educación física -planeación, intervención didáctica, evaluación- son actividades que permiten llevar a cabo los procesos educativos; para la planeación se propone la elaboración de unidades didácticas, que se pueden considerar como unidades de tiempo o subconjuntos del programa anual, un grupo de clases que permiten determinados aprendizajes. Se presentan orientaciones didácticas para que los profesores tengan un referente que les permita la construcción de sus intervenciones didácticas. La evaluación se explica como un proceso formativo permanente, que permite 
corroborar los avances y comprobación de que los educandos logran los aprendizajes esperados.

\section{La salud como tema transversal en Educación Física}

La salud cuenta con un tratamiento transversal en el currículo, ya que, al ser multifactorial, requiere de la intervención de varias asignaturas como Conocimiento del Medio, Ciencias Naturales y Tecnología, Biología, Formación Cívica y Ética, Educación Socioemocional. El área de EF es considerada una de las idóneas para la promoción de la salud, dadas las características de sus contenidos, como la práctica de actividades físicas, promoción del autocuidado, prevención y desarrollo de hábitos de salud, los que permitirán una mejor calidad de vida.

\section{Formación docente}

\section{Transformación de la práctica pedagógica}

El programa menciona que existe una cultura pedagógica tradicional que se basa en exposición de temas por parte de los docentes, a pesar de que la ciencia e investigaciones han demostrado que se limitan los aprendizajes significativos y el pensamiento crítico de los estudiantes, por ello se considera clave se cambie por una cultura pedagógica en la que sean activos, creativos y con aprendizajes acordes con el contexto social actual y para ello es fundamental la participación de los docentes desde su formación inicial y continua.

\section{Formación inicial docente}

En las escuelas formadoras de docentes deberán alinear y ajustar su currículo al de la educación básica para que sea un pilar en la formación de los futuros maestros, así como desarrollar grupos de investigación entre las diferentes instituciones que fortalezcan las tareas educativas. Los maestros que deseen ingresar a trabajar en la educación básica deben dominar el nuevo currículo, ya que deberán presentar un examen diseñado con base en perfiles, parámetros e indicadores debidamente establecidos.

\section{Formación continua de maestros en servicio}

Para que la reforma educativa tenga éxito, se reconoce que los maestros juegan un papel preponderante, por esto, la actualización es considerada esencial; para ello se considera ofertar programas y cursos que atiendan los dominios del perfil docente. Pero además los profesores deberán presentar una evaluación del desempeño, al menos cada cuatro años, por lo que se tendrán que preparar para dichos proceso evaluativos. 


\section{Discusión}

\section{Currículos tradicionales}

Este cambio de programa puede ser una extraordinaria oportunidad para que los educadores físicos optaran por estilos de enseñanza menos directivos y que permitan la participación activa de los alumnos, lo que representa un desafío por lograr, una intervención didáctica en sus sesiones que contemplen un horizonte pedagógico, establecido claramente en los programas, pero que en la realidad no es observado. En varios trabajos se mencionan estos temas; García y Baena-Extremera (2017) expresan que en EF es ineludible poder identificar y distinguir las diferentes metodologías y que en su formación continua los docentes analicen y puedan aplicar, para que los alumnos cambien la percepción de la asignatura, y que no sea la que tradicionalmente conciben. Figueras, Capllonch, Blázquez \& Monzonís (2016) afirman que a partir de que se incluyen los currículos de competencias, hay un interés por las investigaciones reflexivas y críticas, por ejemplo: sobre la necesidad y congruencia de una competencia motriz entre las competencias clave; la adaptación al modelo competencial en el ámbito educativo; profundizar en los aspectos que garantizan la calidad y excelencia del enfoque competencial y, últimamente, la interpretación y percepción de los docentes de EF en relación con las competencias a su desarrollo curricular.

\section{Complejidad}

Frade (2016) dice que lo actores educativos tienen problemas para que las reformas curriculares que desarrollan competencias puedan conceptualizar la educación desde la complejidad, abandonando la disciplina simplista, por lo que el diseño curricular crea gran confusión para la intervención docente. Para cumplir la complejidad en el diseño de planes y programas, las competencias deben ser transdisciplinares, en que los educandos construyan sus aprendizajesy conocimientos, para que sean propositivos, creativos e innovadores y que los docentes tomen en cuenta sus intereses.

Jess, Atencio \& Thorburn (2017) dicen en su estudio que una realidad en EF es que los actores educativos siguen utilizando los modelos conductistas, en lugar de los enfoques complejos, ya que tienen dificultades para actualizarse y ser facilitadores de aprendizajes significativos en actividades que involucren análisis, resolución de problemas y pensamiento libre, con actividades pedagógicas y didácticas flexibles donde los alumnos tengan la posibilidad de auto organizarse, como en una sociedad sumamente compleja, exigente y cambiante; aunque también mencionan que no es posible caer en 
una dicotomía de enfoques buenos y malos, pero sí es urgente producir un cambio hacia modelos de aprendizajes colaborativo y reflexivo, con enfoque modernos y actuales.

\section{Enfoque pedagógico}

En cuanto a la intervención pedagógica de la EF, Castañer \& Camerino (2013) desarrollan un enfoque que busca una comprensión global, fundamentada en el enfoque sistémico de las ciencias humanas y que permite una mejor comprensión de la complejidad trasladada a la motricidad, que permite que los estudiantes interactúen con procesos de análisis y síntesis para entender sus propias actuaciones, permitiéndoles reconfigurarlas y reorganizarlas. Utilizan el símbolo del molino de viento en movimiento, donde el centro es la acción motriz que es el dinamismo y se expande en cuatro dimensiones, o elementos de las capacidades motrices: perceptivo-motrices, físico-motrices, socio-motrices y recreativo-motrices, donde el giro del molino genera interacción entre ellas, lo que permite mayor riqueza a la motricidad y corporeidad de los estudiantes.

La interacción de las capacidades, habilidades y destrezas que promueve la EF, acorde con el enfoque sistémico de la motricidad el programa actual, dice que el desarrollo de habilidades y destrezas se logra poniendo en práctica sus capacidades perceptivo-motrices, socio-motrices, y físico-motrices; hay que decir que el Programa EF (2011) se manejaba para favorecer la motricidad, la corporeidad y la creatividad de los docentes, para que programaran sus actividades con los patrones básicos de movimiento de locomoción, estabilidad y manipulación, con la combinación entre ellos, pudiendo ser sencillas y complejas, lo que permite una mejor base motriz para los educandos.

Respecto de la planeación y evaluación, al hacer revisiones de las prácticas de las acciones motrices se reconoce la importancia del papel de los profesionales de la EF en el desarrollo de competencias de los educandos y, aunque se recalca que la planeación ayuda, por ejemplo, a determinar el inicio y final de unidades didácticas, determinar contendidos, metodologías y evaluaciones, en la realidad no siempre es así. En estudios acerca del tema, Baena \& Granero (2015) en su trabajo sobre orientaciones de la planificación, dicen que son pocas las horas lectivas de EF, por ello se debe reflexionar y planificar sobre los contenidos y la mejor forma de hacerlo por parte del docente y de los alumnos que reciben los contenidos. Una buena planificación permite una mejor docencia, una enseñanza eficaz y calidad en el aprendizaje, así como una más fácil evaluación. Ruiz (2015) menciona en su estudio sobre estilos de enseñanza en EF, que los docentes piensan que pierden el control de sus actividades y que los alumnos no realizarían las actividades de acuerdo 
con sus conceptualizaciones, por lo que los condicionan constantemente con bajar sus notas o suspensiones; pero, cuanto más se esfuerzan en controlar, menos consiguen y los educandos menos responden. Afirma que, probablemente, disminuyendo el control sobre los estudiantes, ellos se sentirían más motivados para participar en sus actividades.

La salud es un tema transversal en Educación Física, en que los adultos pretenden orientar las motivaciones para realizar actividad física en los alumnos hacia la salud y reforzar su carácter, mientras que ellos lo hacen hacia la diversión y el juego. En este caso es aconsejable encontrar el equilibrio que permita desarrollar hábitos de vida. En estudios del tema, Delgado \& Tercedor (2002) dicen que, aunque se considera la salud como tema transversal, también se puede incluir este tema en la asignatura de EF y considerar como eje de las unidades didácticas, incluyendolo en las sesiones clase. Caballero (2015) en su trabajo sobre programas de salud hace dos recomendaciones: implicar y motivar a la comunidad en participar colectivamente, realizando una evaluación inicial de las necesidades y, actualizar a los docentes y dar un seguimiento y, posteriormente, analizar la veracidad de la implantación, proceso y resultados, para poder analizar globalmente.

\section{Formación docente}

Con respecto a la formación inicial y actualización en activo, podemos decir que las prácticas educativas de los docentes de EF tienen una estrecha relación con estos dos aspectos. Primero, se debe considerar la formación inicial en el contexto socioeducativo de la docencia. De ahí que las escuelas formadoras de docentes son fundamentales en el futuro profesional de la educación física. Si en el proceso de formación los futuros educadores utilizan enfoques conductistas en lugar de los enfoques que se exigen en los currículos con los que se trabajará posteriormente, surge la contradicción en la que los futuros docentes tendrán dificultades de interpretación y ejecución de los nuevos modelos educativos.

La profesionalización docente comienza desde el momento en que el educador egresa de una escuela formadora e inicia su trabajo, por lo tanto, la formación inicial y la educación continua son dos procesos que tienen una relación importante.

Se requiere que las autoridades educativas realicen los ajustes necesarios para que los futuros maestros, primero, en la formación inicial, interpreten y apliquen los programas del nuevo modelo educativo $y$, en segundo término, la formación en activo, lo que les permitirá actualizarse para una mejor labor docente en EF. Martínez, Castillo \& Granda (2017) dicen que la práctica docente debe ser 
vista en dos aspectos, la enseñanza recibida, propia de cualquier proceso formativo, y la apropiación del oficio docente, consistente en cómo iniciarse, perfeccionarse y/o actualizarse en la práctica de enseñar. Pastor, Brunicardi, Arribas \& Aguado (2016) indican que, dentro de los retos que tiene la EF en este siglo, la formación inicial e incluso la permanente, basada en competencias, se debe fundamentar en modelos de aprendizaje que desarrollen capacidades cognitivas complejas, con un enfoque competencial y práctico.

En estudios realizados acerca de la formación docente, González, Marín, \& Soto (2019), los resultados indican que, la construcción de la identidad profesional de los docentes es un proceso continuo que involucra varios factores internos como rasgos de personalidad, sentimientos, valores profesionales y el contexto en el que influyen dos aspectos: la elección profesional y las interacciones sociales; que se debe reforzar la eficiencia del proceso enseñanza-aprendizaje con la idea de no solo formar buenos profesionistas, sino desarrollar la identidad que permita generar transformaciones. Tobar, Gaete, Lara, Pérez, \& Freundt (2019), en su trabajo aseveran que las instituciones formadoras de docentes de EF deben promover sus visones, modelos formativos y teorías en los procesos formativos, junto con adaptar los planes y programas a los requerimientos del contexto de la profesión. Obregón, Martínez, Cresp, Arellano \& Vargas (2015) en su estudio buscaron conocer cuáles y cómo evolucionan, en su formación inicial, aspectos como concepciones previas o teorías; en algunos de sus resultados dicen que el estudiantado concede gran importancia a las teorías de la salud.

Por ello, es importante la transformación e implementación de nuevas políticas que tengan impacto en la función docente, que se llevarán a efecto en las canchas escolares y en el aprendizaje de los alumnos. La formación de los futuros docentes debe ser vista de forma integral, en la que se construya un aprendizaje crítico de la teoría, metodología y práctica, para luego situarse en el terreno profesional (Barrera \& Hinojosa, 2017).

\section{Formación continua}

Otro aspecto a considerar en la implementación del nuevo modelo educativo, la formación continua y superación profesional, debe ajustarse a los grandes cambios educativos y la influencia de la tecnología a nivel global, en que los maestros participen activamente, apropiándose de los nuevos enfoques educativos, a través de procesos de actualización y superación profesional. Los procesos de capacitación en nuestro país, en ocasiones, no contemplan las necesidades reales de los docentes, pero lo incongruente es que no respetan los 
preceptos pedagógicos de planes y programas, siendo diseñados cursos y talleres con enfoques tradicionales y alejados de las nuevas tecnologías. Lozada (2017) es claro en su propuesta de que los cursos de actualización integren una construcción colectiva de los conocimientos pedagógicos y didácticos, acorde con la enseñanzaaprendizaje de las escuelas. Cerda \& López (2006) ya desde hace tiempo plantean la necesidad de la actualización a partir del hacer y no desde el discurso del deber ser, un diálogo desde la realidad, desde donde surgen las demandas de análisis de la práctica de la enseñanza, para poder realizar transformaciones en ellas.

\section{Propuestas}

Las escuelas formadoras de docentes deben realizar los cambios curriculares para que los estudiantes desarrollen competencias que impliquen fortalecer sus capacidades cognitivas complejas, participando en procesos educativos acordes con las pedagogías actuales.

Asimismo, es importante poner especial atención a las prácticas profesionales de los estudiantes, ya que este es un eslabón entre el proceso formativo y la práctica real y cotidiana, con aprendizajes de la realidad contextual y todas sus tradiciones y costumbres, por ello, se requiere establecer una normativa a cumplir con objetivos claros, seguimiento y evaluación de la actuación de los futuros docentes en las escuelas donde realizan sus prácticas.

Que exista una relación y comunicación entre las escuelas formadoras y las autoridades educativas de la educación básica para un intercambio institucional de planes y programas y, además, la coordinación de las prácticas de los estudiantes, procurando los apoyos necesarios para que, en los planteles escolares, se realice una práctica que promueva las competencias de los estudiantes, instancia en que los supervisores y asesores técnicos y profesores de EF son fundamentales en los niveles de supervisión y asesoría de los futuros profesores.

Los cursos de actualización y superación profesional de los actores educativos deben ajustarse a las necesidades de los profesionales de EF, con contenidos del área que les permitan desarrollar y fortalecer sus competencias, en que su diseño curricular también contemple una pedagogía y didáctica que promueva una actitud crítica y reflexiva.

\section{Conclusiones}

Las reformas educativas hay que verlas como una oportunidad para renovar los procesos educativos, modernizando sus objetivos y estrategias, que permitan ofrecer una educación de calidad que sirva a los estudiantes para desempeñarse en la vida, pero también sería importante considerar 
la opinión de todos los actores educativos para implementar estos cambios. Por ejemplo, habrá resistencia al cambio si no se convoca y consulta a los profesores sobre la pedagogía y didáctica que se va implementar en las aulas.

Es un gran reto lograr que los docentes cambien sus prácticas adoptadas por largo tiempo, en las que reproducen enfoques pedagógicos y didácticos que no son acordes con la sociedad actual, para ello sería importante una capacitación, asesoría, acompañamiento y seguimiento con los docentes en cada escuela.

Una limitante es que las reformas educativas se hacen cada vez con mayor frecuencia, sin dar la posibilidad de intervenir a los actores educativos, que en la realidad, son quienes implementan dichos cambios. Actualmente se presta más atención a lo político o sindical que a los temas educativos. Por esto es importante realizar investigaciones profundas sobre las implicaciones que lleva un cambio de programa; es necesario promover el análisis y debates entre los profesionales de la EF, para que la asignatura efectivamente promueva la educación integral de los educandos.

\section{REFERENCIAS BIBLIOGRÁFICAS}

Baena, A., \& Granero, A. (2015). Efectos de las actividades en la naturaleza en la predicción de la satisfacción de la Educación Física. Retos: nuevas tendencias en educación física, deporte y recreación, 28, 9-14.

Barrera, M., \& Hinojosa, C. (2017). Incidencia del proceso de la práctica profesional en las dimensiones de la formación docente de estudiantes de pedagogía en educación física. Pensamiento Educativo. Revista de Investigación Educacional Latinoamericana, 54(2), 1-15. doi: 10.7764/PEL.54.2.2017.8

Betancur-Agudelo, J. E., López-Ávila, C. R., \& Arcila-Rodríguez, W. O. (2018). El docente de Educación Física y sus prácticas pedagógicas. Revista Latinoamericana de Estudios Educativos, 14(1), 15-32. doi: 10.17151/rlee.2018.14.1.2

Caballero, P. (2015). El desarrollo positivo en los programas de actividad física y el deporte. EmásF: revista digital de educación física, (32), 82-96.

Casanova, H., Diaz-Barriga, A., Rodríguez-Gómez, R., \& Rueda, M. (2017). El modelo educativo 2016: un análisis desde la investigación educativa. Perfiles Educativos, 155, 194-205 
Castañer, M., \& Camerino, O. (2013). Enfoque dinámico e integrado de la motricidad (EDIM). Acción motríz, 71, 5-12.

Cerda, A., \& López, I. (2006). El grupo de aprendizaje entre pares: una posibilidad de favorecer el cambio de la prácticas cotidianas de aula. Formación Continua de Docentes: Un camino para compartir, 33-44.

Coutín, A., Gastélum, G. y Guedea. J. (2018) Problemas actuales de los currículos en la educación física en Iberoamérica. Una revisión sistemática. Revista Ciencias de la Actividad Física, 19(2), 1-9.

Delgado, M., \& Tercedor, P. (2002). Estrategias de intervención en Educación para la Salud desde la Educación Física. España: INDE.

Figueras, S., Capllonch, M., Blázquez, D. \& Monzonís, N. (2016). Competencias básicas y educación física: estudios e investigaciones. Apunts. Educación Física y Deportes, 123, 32-41. doi: https://doi. org/10.5672/apunts.2014-0983. cat.(2016/1).123.04

Frade, L. (2016). Complejidad y competencias: acerca del cambio paradigmático necesario y posible en la educación contemporánea. (Tesis de doctorado) Multiversidad Mundo Real Ed- gar Morín, AC., México.

García, M., \& Baena-Extremera, A. (2017). Motivación en Educación Física a través de diferentes metodologías didácticas. Profesorado Revista de currículum y formación del profesorado, 27(1), 387-402.

González P., Marín, R. \& Soto, Ma. (2019). La identidad profesional en estudiantes y docentes desde el contexto universitario: una revisión. Revista Ciencias de la Actividad Física UCM, 20(1), 1-14. https://doi.org/10.29035/ rcaf.20.1.4

Herrera San Martín, E. (2012). La UVE de Gowin como instrumento de aprendizaje y evaluación de habilidades de indagación en la unidad de fuerza y movimiento. Paradígma, 33(2), 101-126. Recuperado de http://ve.scielo. org/scielo.php?script=sci_ a r t text \& p i d = S 1011 22512012000200006\&lng=es\&t| $\mathrm{ng}=\mathrm{es}$

Jess, M., Atencio, M., \& Thorburn, M (2017). Teoría de la complejidad para informar los desarrollos de la Educación Física en Escocia. Propuesta Educativa, 7(47), 6883. 
Lozada, J. C. P. (2017). Formación Permanente de los Docentes como Referente de la Calidad Educativa. Revista Scientific, 2(5), 125139.

Lucio, R. (1992). La construcción del sabery del saber hacer. Educación y Pedagogía, 8-9. Recuperado de https://aprendeenlinea.udea. edu.co/revistas/index.php/revistaeyp/article/view/5675/5095

Martínez, J. E., Castillo, L. C., \& Granda, V. D. (2017). Formación inicial del docente en educación física y su desempeño profesional. Revista Digital de Educación Física, 48, 83-95.

Obregón, C.; Martínez, C.; Cresp, M.; Arellano, R. \& Vargas, R. (2015). Formación inicial docente, Teorías y Paradigmas en Educación Física. Revista de Ciencias de la Actividad Física UCM, 16(2), 3746. Recuperado de http://revistacaf.ucm.cl/article/view/83

Opertti, R. (2017). 15 claves de análisis para apuntalar la agenda educativa 2030; Cuestiones fundamentales y actuales del currículo y el aprendizaje, 74. https://www.cife.edu.mx/Biblioteca/public/Libros/19/15-clavesapuntalar-agenda-educativa. pdf
Pastor, V. M. L., Brunicardi, D. P., Arribas, J. C. M., \& Aguado, R. M. (2016). Los retos de la Educación Física en el Siglo XXI. Retos: nuevas tendencias en educación física, deporte y recreación, (29), 182-187.

Ruiz, L. (2015). Estilo de enseñanza en educación física y sus consecuencias comportamentales, afectivas y cognitivas (Tesis doctoral) Universidad de Málaga, España.

Secretaría de Educación Pública. (1993). Programas de Educación Física. México: Secretaría de Educación Pública.

Secretaría de Educación Pública. (1988). Programas de Educación Física, Primaria. México: Secretaría de Educación Pública.

Secretaría de Educación Pública. (2011). Programas de Estudio, Educación Básica. México: Secretaría de Educación Pública. Recuperado de https://sector2federal.wordpress.com > plany-programas-de-estudio-2011_ fpdf 
Secretaría de Educación Pública. (2017). Aprendizajes claves para la educación integral. Educación Física. Educación básica Plan y programas de estudio, orientaciones didácticas y sugerencias de evaluación. México: Secretaría de Educación Pública.

Tobar, B. A. U., Gaete, M. J. F., Lara, M. M., Pérez, A. M., \& Freundt, A. R. (2019). Teorías implícitas y modelos de formación subyacentes a la percepción de rol del profesor de educación física. Retos, 36(36), 159-166.
UNESCO. (2017). Herramientas de formación para el Desarrollo Curricular: Desarrollo y Aplicación de Marcos Curriculares. Ginebra, Suiza. Organización de las Naciones Unidas para la Educación, la Ciencia y la Cultura. Recuperado de http://www. ibe.unesco.org/fileadmin/user_ upload/Publications/Training_ tools/IBE-CRP-2014_spa.pdf

\section{Dirección para correspondencia}

Adrián Alonso Ramírez García

Doctor en Ciencias de la Actividad Física.

Facultad de Ciencias de la Actividad Física, UACH - Servicios Educativos del Estado de Chihuahua, México.

Contacto:

dr.adrianramirezgarcia@gmail.com

Recibido: 24-10-2018

Aceptado: 11-10-2019 